\title{
The Impact of Magnification and Size Bias on Weak Lensing Power Spectrum and Peak Statistics
}

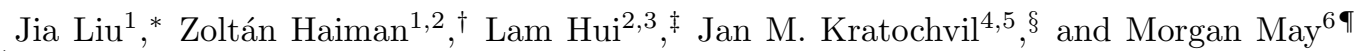 \\ ${ }^{1}$ Department of Astronomy and Astrophysics, Columbia University, New York, NY 10027, USA \\ 2 Institute for Strings, Cosmology, and Astroparticle Physics (ISCAP), \\ Columbia University, New York, NY 10027, USA \\ 3 Department of Physics, Columbia University, New York, NY 10027, USA \\ 4 Department of Physics, University of Miami, Coral Gables, FL 33146, USA \\ ${ }^{5}$ Astrophysics and Cosmology Research Unit, University of KwaZulu-Natal, Westville, Durban, 4000, South Africa and \\ ${ }^{6}$ Physics Department, Brookhaven National Laboratory, Upton, NY 11973, USA
}

(Dated: July 9, 2018)

\begin{abstract}
The weak lensing power spectrum is a powerful tool to probe cosmological parameters. Additionally, lensing peak counts contain cosmological information beyond the power spectrum. Both of these statistics can be affected by the preferential selection of source galaxies in patches of the sky with high magnification, as well as by the dilution in the source galaxy surface density in such regions. If not accounted for, these biases introduce systematic errors for cosmological measurements. Here we quantify these systematic errors, using convergence maps from a suite of ray-tracing Nbody simulations. At the cut-off magnitude $m$ of on-going and planned major weak lensing surveys, the logarithmic slope of the cumulative number counts $s \equiv d \log n(>m) / d \log m$ is in the range $0.1 \lesssim s \lesssim 0.5$. At $s \approx 0.2$, expected in the $I$ band for LSST, the inferred values of $\Omega_{m}, w$ and $\sigma_{8}$ are biased by many $\sigma$ (where $\sigma$ denotes the marginalized error) and therefore the biases will need to be carefully modeled. We also find that the parameters are biased differently in the $\left(\Omega_{m}, w, \sigma_{8}\right)$ parameter space when the power spectrum and when the peak counts are used. In particular, $w$ derived from the power spectrum is less affected than $w$ derived from peak counts, while the opposite is true for the best-constrained combination of $\sigma_{8} \Omega_{m}^{\gamma}$ (with $\gamma=0.62$ from the power spectrum and $\gamma=0.48$ from peak counts). This suggests that the combination of the power spectrum and peak counts can help mitigate the impact of magnification and size biases.
\end{abstract}

PACS numbers: PACS codes: 98.80.-k, 95.36.+x, 95.30.Sf, 98.62.Sb

\section{INTRODUCTION}

By measuring the distortions of background galaxy shapes by foreground masses (galaxies, galaxy clusters, and large-scale structures), weak gravitational lensing (WL) surveys probe the mass density fluctuations throughout the cosmic span (see recent reviews by 1 4). WL observations, in conjunction with cosmological simulations, can be used to place precise constraints on cosmological parameters. Recent WL surveys, such as COSMOS [5] and CFHTLenS [6], have measured the shear power spectrum and have already placed useful constraints on $\Omega_{m}$ (the matter density of the universe), $\sigma_{8}$ (the amplitude of the primordial power spectrum on a scale of $8 h^{-1}$ comoving Mpc), and $w$ (the dark energy equation of state).

Because of the statistical nature of WL surveys, it is important to have an unbiased sample of source galaxies, fairly sampling the foreground density fluctuations across the sky. In this paper, we investigate possible sources of

\footnotetext{
*Electronic address: jia@astro.columbia.edu

$\dagger$ Electronic address: zoltan@astro.columbia.edu

$\ddagger$ Electronic address: Ihui@astro.columbia.edu

$\S$ Electronic address: jank@physics.miami.edu

ๆElectronic address: may@bnl.gov
}

bias in flux-limited surveys, arising from a preferential selection of source galaxies in patches of the sky with high magnification, as well as by the dilution in the source galaxy surface density in such regions (known as magnification bias; hereafter MB). MB has been studied extensively in the past for its impact on galaxy-quasar and galaxy-galaxy correlation functions in 2D [7-15], and in $3 \mathrm{D}$ 16 18, and on the statistics of the Lyman- $\alpha$ forest [19]. An additional size bias (hereafter SB) can be present in surveys in which the selection of the source galaxies depends on their angular sizes. If not accounted for, these biases represent a systematic error for cosmological measurements. In the context of WL, the impact of MB and SB have been studied for the power spectrum [20, 21] and for high peaks caused by individual NFW halos [22].

Ref. 20 has shown that ignoring MB and SB in the shear power spectrum can cause $2-3 \sigma$ deviations in cosmological parameter estimation for a DETF [23] Stage III experiment, such as the Dark Energy Survey. ${ }^{1}$ Future WL surveys with larger sky coverage and/or deeper observations, such as those by planned by the Large Syn-

\footnotetext{
1 http://www.darkenergysurvey.org
} 
optic Survey Telescope ${ }^{2}$ (LSST) and Euclid ${ }^{3}$, will have significantly better statistical sensitivity, and therefore can be more severely impacted by these biases.

In this paper, we first show that MB is indeed significant for the power spectrum, extending earlier results 20 to explicitly compute the biases on cosmological parameters. We then focus on the impact of MB on peak counts. Lensing peaks were first considered as a cosmological probe in early ray-tracing simulations a decade ago [24. Peak counts have received increasing attention in recent years $25-33$ as a way to access cosmological information from the strong non-Gaussianities in the lensing fields. In particular, these studies have shown that the number and height-distribution of peaks have high cosmological sensitivity, and can improve cosmological constraints by a factor of $\sim$ two, compared to using the power spectrum alone.

Peak counts are a simple and robust statistic, defined by recording local maxima in a $2 \mathrm{D}$ shear or convergence ( $\kappa)$ map, smoothed by suitable filters. Ref. 28 investigated the physical origin of the individual $\kappa$ peaks, by tracing their contributing light rays back in time across their N-body simulation boxes. They found that high peaks (with amplitudes $\gtrsim 3.5 \sigma_{\kappa}$, where $\sigma_{\kappa}$ is the r.m.s. of the convergence $\kappa$ ) are typically created by individual massive halos. It has been shown that MB increases the signal-to-noise and therefore the total number of such peaks [22]. By comparison, low peaks $\left(\sim 1-2 \sigma_{\kappa}\right)$ are typically caused by a combination of (cosmologyindependent) shape noise and a (cosmology-dependent) constellation of 4-8 lower-mass halos. These halos have masses of a few $\times 10^{12} \mathrm{M}_{\odot}$, and are offset by $\sim \operatorname{arcmin}$ from the line of sight to the center of the peak. The low peaks are especially promising, as they carry the majority of cosmological information, and are relatively insensitive to baryonic cooling that affect the halo cores [32. We therefore extend the earlier results of [22] for high peaks, where increases in both the peak heights and number of high peaks were seen, to the low peaks, and to explicitly compute the biases on cosmological parameters.

To study the impact of MB, we build a simple numerical model to derive cosmological parameters (and their error bars) using either the power spectrum or peak counts measured in our simulations. We then apply magnification bias to a set of "true" convergence maps (which faithfully represent the projected dark matter distribution in a fiducial flat $\Lambda$ CDM cosmology) to create mock "biased" maps, mimicking an observed dataset. For each of these "biased" datasets, we find the best-fit set of the three cosmological parameters $\left(\Omega_{m}, w\right.$ and $\left.\sigma_{8}\right)$, using the "true" maps for the model fitting. Finally, we quantify the difference between the inferred cosmology and the true fiducial cosmology, as a function of the strength and

\footnotetext{
2 http://www.lsst.org

3 http://sci.esa.int/euclid
}

sign of the magnification and size bias (determined by the slope of the galaxy luminosity function and the galaxy size distribution).

The rest of this paper is organized as follows: in $\S$ II we introduce the formalism of magnification bias, and discuss its principal ingredient, the galaxy luminosity function. We then describe our computation methods in $\S$ III, including the convergence maps created with our ray-tracing N-body simulations, computing the power spectra and the peak distributions from these maps, determining the cosmology-dependence of these quantities, and finally applying biases to the maps to create mock observations. We present our main results in $\S$ IV , where we fit the mock data, and show that $\mathrm{MB}$ and $\mathrm{SB}$ will indeed alter the derived cosmological parameters by many $\sigma$. Finally, in $\S \mathrm{VI}$, we summarize our conclusions and the implications of this work.

\section{MAGNIFICATION BIAS}

Gravitational lensing causes a bias by modulating the apparent surface density of galaxies on the sky, through two competing effects [34. First, lensing can magnify (or demagnify) individual source galaxies in the background, increasing (or decreasing) their total flux. In a flux-limited WL surveys, some otherwise excluded faint galaxies can therefore make it into (or drop out of) the sample because of this (de)magnification. Second, a similar (de)magnification applies to the patch of the sky around the galaxy, geometrically diluting (or enhancing) the apparent surface density of galaxies in this region. These two effects counteract each other, and the net bias depends on the slope of the intrinsic (unlensed) galaxy luminosity function at the survey flux limit. In addition to these effects, lensing can increase (or decrease) the apparent angular size of spatially resolved individual galaxies. If either the survey selection, or a derived statistic such as WL shear, depends on the apparent size, then this can introduce an additional size bias.

\section{A. Formalism}

To quantify the effect of MB, we follow the discussion in Appendix A of [17. Including the effect of lensing on both the flux and on the geometrical surface density, we have the relation

$$
n(\boldsymbol{\theta})=n_{g}(\boldsymbol{\theta})[1+(5 s-2) \kappa(\boldsymbol{\theta})],
$$

where $n(\boldsymbol{\theta})$ is the observed (lensed) galaxy number density at position $\boldsymbol{\theta}$, as viewed by the observer, $n_{g}(\boldsymbol{\theta})$ is the intrinsic (unlensed) galaxy number density, $s$ is the slope of the cumulative number counts evaluated at $m_{\lim }$, and $\kappa(\boldsymbol{\theta})$ is the convergence. This equation assumes the weak lensing limit $(\kappa \ll 1)$, neglects the correspondingly small difference $\delta \boldsymbol{\theta}$ between lensed and unlensed directions on 
the sky, and also assumes that galaxy number density fluctuations $\delta n_{g} / n_{g}$ on the angular scales of interest are small, as well. Under these assumptions, the above equation is valid to first order in $\kappa, \delta \boldsymbol{\theta}$, and $\delta n_{g}$. Finally, if we assume a survey with a sharp magnitude cut-off at $m_{\text {lim }}$ :

$$
s=\left.\frac{\partial \log _{10} n_{g}}{\partial m}\right|_{m_{\mathrm{lim}}} .
$$

\section{B. Galaxy Luminosity Functions and WL Surveys}

The magnitude of MB depends on the galaxy luminosity function through the logarithmic slope $s$. Observed luminosity functions are well described by a Schechter function 35,

$$
\begin{aligned}
\Phi(M) d M & =(0.4 \ln 10) \Phi^{\star}\left[10^{-0.4\left(M-M^{\star}\right)}\right]^{\alpha+1} \\
& \times \exp \left[-10^{-0.4\left(M-M^{\star}\right)}\right] d M
\end{aligned}
$$

where $\Phi(M) d M$ is the number density of galaxies with magnitude between $M$ and $M+d M, \Phi^{\star}$ is a characteristic number density (in $\mathrm{Mpc}^{-3}$ ), and $M^{\star}$ is a characteristic magnitude. It consists of a power-law with index $\alpha$ at the faint end and a exponential cut-off at the bright end. The cumulative galaxy number density can be written as

$$
n_{g}=\int_{-\infty}^{M_{l i m}} \Phi(M) d M
$$

Note that this equation holds at a given redshift. In Fig. 1. we show $s$ calculated using equation (2), as a function of cut-off magnitudes in the G, R, I and $\mathrm{Z}$ bands at redshifts $z=0.5$ and $z=1$. In this calculation, we used the measurements of $\Phi^{\star}, M^{\star}$ and $\alpha$ by [36, 37, which are all redshift-dependent. ${ }^{4}$

Table 1 lists the magnitude limits and the corresponding values of $s$ for several current and future WL surveys. We note that in order to measure the shape of the galaxies, it is necessary to adopt a brighter magnitude than for the point sources. For surveys where the magnitude limit was available only for point sources, we adopted a one magnitude brighter value for $m_{\mathrm{lim}}$. For simplicity, for broad multi-band filters $(\mathrm{R}+\mathrm{I}+\mathrm{Z})$, we have calculated $s$ using the central I band. Table I shows that surveys with $m_{\text {lim }, I} \approx 24-25$ have $s \approx 0.2$, assuming a mean redshift $z=0.5$. While the effect of MB almost disappears $(s \approx 0.4)$ for galaxies at $z=1.0$. Ref. 20] has shown that the $z$-dependence is much weaker than the $s$-dependence. For LSST, we expect the effective galaxy

\footnotetext{
${ }^{4}$ Eq.1 in ref. 36] describes the redshift evolution of $\Phi^{\star}, M^{\star}$ and $\alpha$. The parameters can be found in table $3 \& 4$ of ref. [36] for the G band, and in table 9 ("case 3", with a constant $\alpha=-1.33$ ) of ref. 37] for the R, I, Z bands.
}
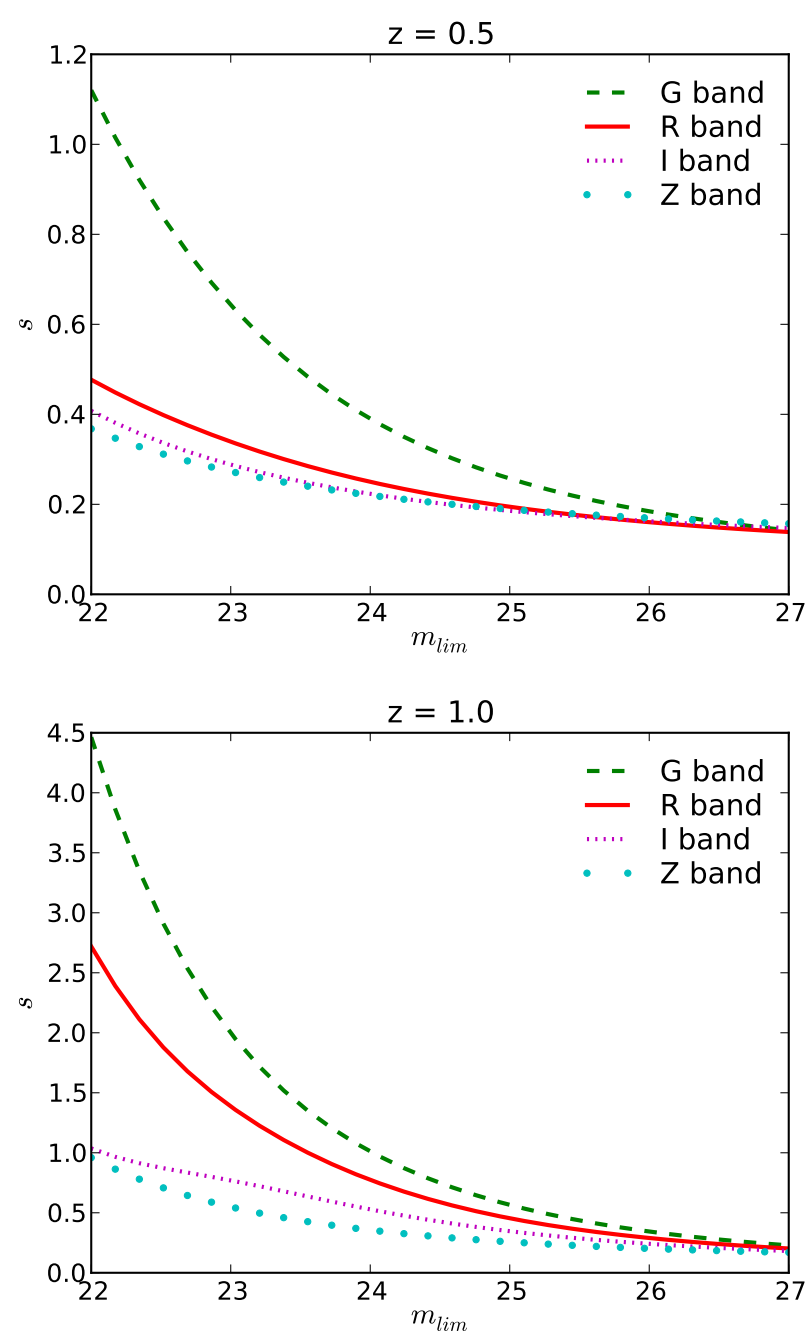

FIG. 1: The logarithmic slope $s$ of the galaxy number counts as a function of cut-off magnitude $m_{\mathrm{lim}}$ at two different redshifts $z=0.5$ (top) and $z=1$ (bottom). Four different filters $(\mathrm{G}, \mathrm{R}, \mathrm{I}, \mathrm{Z})$ are shown. WL surveys target a depth of $m_{\text {lim }}>24$ to achieve a sufficiently large galaxy number density. As a result, the relevant range of $s$ is $0.1 \lesssim s \lesssim 0.6$ (see Table I).

number density after applying lensing cuts to peak at a lower redshift $(z=0.5-0.8)$ than the raw sample $(z \gtrsim 1.0)$. To illustrate the effect of MB, we adopt $s=0.2$ as our fiducial value, corresponding to $z=0.5$ for the conservative cut (see Figure 7 in ref. [38]). We will show in $\S \mathrm{IV}$ that MB will significantly affect the power spectrum and the peak counts at this value.

\section{Size Bias}

If a survey has a cut in galaxy size $r$, in addition to a flux cut, then equation (1) is modified to

$$
n(\boldsymbol{\theta})=n_{g}(\boldsymbol{\theta})[1+(5 s+\beta-2) \kappa(\boldsymbol{\theta})],
$$




\begin{tabular}{|c|c|c|c|c|}
\hline & \multicolumn{4}{|c|}{ Magnitude limit $s(z=0.5) s(z=1.0)$} \\
\hline LSST & $\mathrm{I} \leq 24.8$ & 0.19 & 0.38 & 39 \\
\hline Euclid & $\mathrm{R}+\mathrm{I}+\mathrm{Z} \leq 24.5$ & 0.20 & 0.43 & 40 \\
\hline COSMOS & $\mathrm{I} \leq 25$ & 0.19 & 0.35 & 41 \\
\hline CFHTLS & $\mathrm{I} \leq 24.7$ & 0.19 & 0.39 & 42 \\
\hline DES & $\mathrm{I} \leq 24.3$ & 0.21 & 0.46 & 43 \\
\hline DUNE & $\mathrm{R}+\mathrm{I}+\mathrm{Z} \leq 24.5$ & 0.20 & 0.43 & 44 \\
\hline $\mathrm{KiDS}$ & $\mathrm{R} \leq 25.2(*)$ & 0.24 & 0.69 & 45 \\
\hline $\mathrm{HSC}$ & $\mathrm{I} \leq 26.2\left(^{*}\right)$ & 0.18 & 0.32 & 46 \\
\hline
\end{tabular}

TABLE I: Magnitude limits and corresponding $s$ (number count slope) at $z=0.5$ and 1.0 for current and future WL surveys. For surveys in which only the point source magnitude limit was available (marked by a "*"), we reduced $m_{\lim }$ by 1 magnitude to represent an extended source magnitude cut. In the broad multi-band $(\mathrm{R}+\mathrm{I}+\mathrm{Z})$, we calculated $s$ for the central I band.

where in the case of a sharp cut, the new term $\beta$ is the logarithmic slope of the galaxy size distribution,

$$
\beta=-\left.\frac{\partial \ln n_{g}}{\partial \ln r}\right|_{r_{\mathrm{lim}}} .
$$

This equation assumes that the size and flux cuts are independent, and also that the slopes $s$ and $\beta$ only weakly depend on $r$ and $M$. Under these simple assumptions, the effects of size and magnification bias are equivalent, and only the combination $(5 s+\beta)$ matters. A more sophisticated treatment will eventually be necessary (and will depend on the details of the survey, including how galaxy sizes affect measurement errors). Here we simply note that at the limiting magnitudes of $m_{\text {lim }} \approx 24-25$, the observed angular size distribution has a slope of $\beta \sim 3$ [21. Therefore the additional effect of size bias is equivalent to increasing the value of $s$ by 0.6 , i.e. the relevant fiducial value for LSST with a flux + size cut is changed from $s \approx 0.2$ to $s \approx 0.8$. This means that the sign of the effect changes when we add size bias to magnification bias, as the effect of galaxy density dilution dominates over individual galaxy magnification.

\section{METHODOLOGY}

\section{N-body Simulations}

The N-body simulations and lensing maps were created with the Inspector Gadget lensing simulation pipeline on the New York Blue IBM BlueGene supercomputer. The N-body simulations are the same as the ones used in our earlier work [27, 28, 30, 32, 33. We refer readers to these papers for more detailed information. Here we briefly describe the basis of the simulations and the parameters used.

This work uses in total 35 different N-body simulations, covering 7 different cosmological models (1 fiducial cosmology plus 6 variations), each with 5 independent realizations of the same input primordial power spectrum. We chose our fiducial cosmological model to be

\begin{tabular}{l|c|c|c}
\hline & $\sigma_{8}$ & $w$ & $\Omega_{m}$ \\
\hline Fiducial & 0.798 & -1.0 & 0.26 \\
High- $\sigma_{8}$ & 0.850 & -1.0 & 0.26 \\
Low- $\sigma_{8}$ & 0.750 & -1.0 & 0.26 \\
High- $w$ & 0.798 & -0.8 & 0.26 \\
Low- $w$ & 0.798 & -1.2 & 0.26 \\
High- $\Omega_{m}$ & 0.798 & -1.0 & 0.29 \\
Low- $\Omega_{m}$ & 0.798 & -1.0 & 0.23 \\
\hline
\end{tabular}

TABLE II: Cosmological parameters in each model. The universe is assumed to be spatially flat $\left(\Omega_{\Lambda}+\Omega_{m}=1\right)$.

$\Omega_{m}=0.26, w=-1.0$, Hubble constant $H_{0}=0.72$, with a primordial matter power spectrum with $\sigma_{8}=0.798$ and a spectral index of $n_{s}=0.96$, using the best fit values from the seven-year results by the WMAP satellite [47. We vary each of the 3 parameters $\left(\Omega_{m}, w\right.$ and $\left.\sigma_{8}\right)$ one at a time (a higher value and a lower value than in the fiducial model), while keeping the other 2 parameters at the fiducial values. The 6 non-fiducial models have values of $\Omega_{m}=\{0.23,0.29\}$ (while $\Omega_{\Lambda}=\{0.77,0.71\}$ to keep a spatially flat universe), $w=\{-1.2,-0.8\}$ and $\sigma_{8}=\{0.75,0.85\}$. The combinations are listed in Table I

The N-body simulations were generated using a modified version of the Gadget-2 $\operatorname{code}^{5}$, and they consist of dark matter only. Each run has a box size of $240 h^{-1}$ comoving Mpc, containing $512^{3}$ particles. This corresponds to a mass resolution of $7.4 \times 10^{9} h^{-1} M_{\odot}$. The initial (linear) total matter power spectrum was computed with the Einstein-Boltzmann code $\mathrm{CAMB}^{6}[48$ at $z=0$ and scaled back to $z=100$, which is the starting point of our simulations. The power spectrum was then fed into $\mathrm{N}-\mathrm{GenIC}$, the initial condition generator associated with Gadget2.

\section{Ray-Tracing and Lensing Maps}

To construct convergence maps, we perform raytracing. First, we output 3D boxes at redshifts corresponding to every $\sim 80 \mathrm{Mpc}$ (comoving). We then divide the 3D box into many parallel pieces and project each slice onto a 2D plane perpendicular to the observer's line of sight, using the the triangular shaped cloud (TSC) scheme [49]. In the next step, we convert the surface density to gravitational potential at each plane using Poisson's equation. Each 2D plane has a resolution of $4096 \times 4096$ pixels. We then follow $2048 \times 2048$ light rays from $z=0$, traveling backward through the projection planes. The deflection angle and WL convergence and shear are calculated at each plane for each light ray. These depend on the first and second derivatives of the

\footnotetext{
5 http://www.mpa-garching.mpg.de/gadget/

6 http://camb.info/
} 
gravitational potential, respectively. Between the planes, the light rays travel in straight lines. Finally, for each of the 7 cosmological models, we create 1,000 convergence maps of $12 \mathrm{deg}^{2}$ each in size. This is done by mixing simulations of different realizations, and randomly rotating and shifting the simulation data cubes.

We add galaxy ellipticity noise to our maps, due to variations in the intrinsic shapes of galaxies, and their random orientations on the sky. This shape noise is added to the raw convergence maps using a redshiftdependent expression for the noise in one component of the shear [50]:

$$
\sigma_{\lambda}(z)=0.15+0.035 z
$$

For each pixel, we add $\kappa_{\text {noise }}$ drawn from a random Gaussian distribution centered at zero with variance [51]

$$
\sigma_{\text {noise }}^{2}=\frac{\left\langle\sigma_{\lambda}^{2}\right\rangle}{n_{\text {gal }} \Delta \Omega}
$$

where $n_{\text {gal }}$ is the number of galaxies per $\operatorname{arcmin}^{2}$, and $\Delta \Omega$ is the solid angle of a pixel in units of $\operatorname{arcmin}^{2}$. In the case of LSST, we expect $n_{\text {gal }} \sim 30 \operatorname{arcmin}^{2}[38$ for galaxies that are usable for shape measurements, and it follows that $\sigma_{\text {noise }}=0.33$. This is much larger than the WL signal, whose r.m.s. value (at $z=1$ ) for noise-free maps is $\sigma_{\kappa}=0.02$. To average out the random galaxy noise, we perform smoothing on individual maps with a Gaussian kernel:

$$
\begin{aligned}
\kappa_{G}\left(\boldsymbol{\theta}_{\mathbf{0}}\right) & =\int d^{2} \theta W_{G}\left(\left|\boldsymbol{\theta}-\boldsymbol{\theta}_{\mathbf{0}}\right|\right) \kappa(\boldsymbol{\theta}) \\
W_{G}(\theta) & =\frac{1}{2 \pi \theta_{G}^{2}} \exp \left(-\frac{\theta^{2}}{2 \theta_{G}^{2}}\right),
\end{aligned}
$$

where $\kappa_{G}$ is the smoothed $\kappa$ value at pixel $\theta_{0}$ and $W_{G}$ is the Gaussian kernel with a smoothing scale $\theta_{G}=1 / \sqrt{2}$ arcmin. $^{7}$ The choice of smoothing scale has a known effect on the total peak counts and the shape of the peak distribution. Increasing the smoothing scale generally reduces the total number of peaks and increases the width of the distribution. It has been shown that smaller smoothing scales $(\sim 1 / \sqrt{2}$ arcmin) generally give better constraints, and also that combining a few different scales can further improve the errors [29, 30]. Finding the range of optimal smoothing scales and filter shapes will have to be done specifically for each survey with different characteristics. In this paper, we continue to use the single smoothing scale $\theta_{G}=1 / \sqrt{2}$ arcmin for simplicity, and to facilitate comparison with previous works.

\footnotetext{
${ }^{7}$ We note that in previous papers of this series [27, 28, 30, 32, a different definition of $W_{G}(\phi)=\frac{1}{\pi \theta_{G}^{2}} \exp \left(-\frac{\phi^{2}}{\theta_{G}^{2}}\right)$ was adopted. When using the more commonly used definition $W_{G}$ (eq. 10 , our smoothing scale of $\theta_{G}=1 / \sqrt{2}$ arcmin is equivalent to their $\theta_{G}=1$ arcmin.
}

For simplicity, we use only convergence maps for source galaxies at the single redshift $z=1$, as the $z$-dependence of MB has shown to be weak [20]. Future work should employ tomography with multiple redshifts, and fold into the analysis the actual $z$-distribution of the source galaxies. In total, we have 7,000 convergence maps; we call these the "true" maps, since they do not include any magnification bias. We use this set of maps to predict the cosmology-dependent observables (power spectra or peak counts), which will be described in detail in $\S$ III 5 .

\section{Power Spectra and Peak Counts}

The power spectrum is the most widely used statistic in current WL surveys, and has already been shown to be affected significantly by $\mathrm{MB}$ 20]. We revisit the impact of $\mathrm{MB}$ on the power spectrum in order to crosscheck our simulation results, and to explicitly compute the resulting biases on the cosmological parameters.

We first compute the power spectra for spherical harmonic index $\ell$ in the range $100<\ell<100,000$, with 1000 equally spaced (linear) bins. This covers the range of angles from our pixel size $(\sim 6$ arcsec $)$ to the linear size of our maps $(\sim 3.5 \mathrm{deg})$. In our previous work [27, 30] we compared our numerical power spectrum with the semianalytical power spectrum obtained using the Limber approximation [52] and integrating the nonlinear 3D matter power spectrum along the line of sight [53. Our power spectrum loses power on large scales below $\ell \sim 400$ due to our finite box size, and on small scales above $\ell \sim 20,000$ due to spatial resolution; there is excellent agreement with the semi-analytic predictions between these scales.

Peak counting is done by simply scanning through the pixels on a convergence map, and identifying local maxima (i.e. pixels with a higher value of $\kappa$ than its surrounding 8 pixels). We then record the number of peaks as a function of their central $\kappa$ value.

\section{Applying Bias to the Convergence Maps}

On each of the 1000 maps in our fiducial cosmology, we apply different levels of $\mathrm{MB}$, ranging from $s=-0.5$ to 1.0 , with a step size $\Delta s=0.01$. To do this, on each fiducial map, we take into account the $(5 s-2) \kappa$ factor in eq. (1) and add $\kappa_{\text {noise }}$ when smoothing the map. Eq. (9) becomes (with $\boldsymbol{\theta}$ dependence suppressed for $\kappa$ and $\left.\kappa_{\text {noise }}\right)$ :

$$
\kappa_{G}=\frac{\int d^{2} \theta W_{G}\left[(1+(5 s-2) \kappa) \kappa+\kappa_{n o i s e}\right]}{\int d^{2} \theta W_{G}[1+(5 s-2) \kappa]} .
$$

This is the smoothed $\kappa$ at each pixel, weighted by the galaxy number densities modified by MB. Note that we assume the intrinsic (unlensed) galaxy number density to be a constant - this ignores the effects of shot noise arising from a discrete sampling of the $\kappa$ field by a finite 
number of galaxies, as well as the clustering of galaxies. Other than applying MB, the same procedures are then followed to add noise, smooth the maps, count peaks, or compute power spectra, on the "bias" maps, as for the "true" maps.

\section{Predictions in Other Cosmologies}

In this subsection, we describe how we interpolate (and extrapolate) the peak counts and power spectra for other cosmologies, using our set of simulations in the 7 different cosmologies listed in Table II.

First, for individual convergence maps, we histogram the $\kappa$ peaks into 200 equally spaced bins ranging from $\kappa=-0.02$ to 0.19 (this choice for the number of bins will be justified in $\S \mathrm{V}$ (vii) below). We then calculate the mean peak distribution (average of the 1,000 maps) in each of the 7 cosmology models. To predict the peak distribution for an arbitrary combination of cosmological parameters, we treat each $\kappa$ bin individually, and use a Taylor expansion:

$$
\begin{aligned}
\overline{N_{i}}\left(\Omega_{m}, w, \sigma_{8}\right) & =\overline{N_{i}}\left(\Omega_{m}^{\star}, w^{\star}, \sigma_{8}^{\star}\right) \\
& +\frac{\partial \overline{N_{i}}}{\partial \Omega_{m}} \Delta \Omega_{m}+\frac{\partial \overline{N_{i}}}{\partial w} \Delta w+\frac{\partial \overline{N_{i}}}{\partial \sigma_{8}} \Delta \sigma_{8}
\end{aligned}
$$

Here $\overline{N_{i}}$ denotes the total number of peaks in the $i^{\text {th }}$ bin $(i=1,2 \ldots 200)$, averaged over 1000 maps. $\Delta \Omega_{m}, \Delta w$ and $\Delta \sigma_{8}$ are the differences of the desired parameters $\left(\Omega_{m}, w, \sigma_{8}\right)$ from the fiducial parameters $\left(\Omega_{m}^{*}, w^{*}, \sigma_{8}^{*}\right)$.

The same method was followed for the power spectrum, by simply replacing the peak counts $N_{i}$ with $P_{i}=P\left(\ell_{i}\right)$, the total power in the $i^{t h} \ell$ bin.

In the body of our paper below, we chose to use the fiducial and the "high" models as defined in Table II to compute the cosmology derivatives in equation 12 by a simple finite difference. We call these "forward derivatives". Given that we also have "low" models for each parameter, ideally we could use all three models to refine these predictions, either by including second-order terms in the Taylor expansion, or using two-sided linear derivatives. In practice, we chose to avoid a secondorder expansion, in order to be able to perform an analytical $\chi^{2}$ minimization (see next subsection). We have attempted to use a two-sided derivative, but have found that this caused numerical problems (the discontinuity in the derivative can cause the fitting procedure, described below, to become stuck). We therefore use the forward derivatives in the bulk of this paper. We will discuss the differences in our results if we use "backward derivatives" instead in $\S \mathrm{V}$.

\section{Finding the Best-Fit Cosmology}

To fit a cosmology to one of our biased maps (or more generally to an arbitrary peak count distribution), we minimize a $\chi^{2}$, defined as

$$
\chi^{2}\left(\Omega_{m}, w, \sigma_{8}\right)=\Delta N_{i} C_{i j}^{-1} \Delta N_{j} .
$$

Here $\Delta N_{i}=N_{i}^{\prime}-\overline{N_{i}}\left(\Omega_{m}, w, \sigma_{8}\right)$ is the difference between the peak distribution in a given single map $\left(N_{i}^{\prime}\right)$ and the model $\left(\overline{N_{i}}\right)$ in the $i^{\text {th }}$ bin, and $C_{i j}^{-1}$ is the unbiased estimator of the inverse covariance matrix [54, 55. Summation is implied over repeated indices $i, j$. We make the simple assumption that the peak counts depend linearly on the three parameters. It then becomes possible to write down an analytical solution to the best fitted parameters. By defining

$$
\begin{aligned}
X_{i \alpha} & =\frac{\partial \overline{N_{i}}}{\partial p_{\alpha}} \\
Y_{i} & =N_{i}^{\prime}-\overline{N_{i}}\left(\Omega_{m}^{\star}, w^{\star}, \sigma_{8}^{\star}\right),
\end{aligned}
$$

where $p_{\alpha}=\left(\Omega_{m}, w, \sigma_{8}\right)$ is a three-component vector and $\alpha=1,2,3$ denotes one of the three parameters, we can rewrite

$$
\begin{aligned}
\Delta N_{i} & =Y_{i}-X_{i \alpha} d p_{\alpha} \\
\chi^{2} & =\left(Y_{i}-X_{i \alpha} d p_{\alpha}\right) C_{i j}^{-1}\left(Y_{j}-X_{j \beta} d p_{\beta}\right)
\end{aligned}
$$

Setting $d \chi^{2} / d\left(d p_{\alpha}\right)=0$, we obtain

$$
\begin{aligned}
& X_{i \alpha} C_{i j}^{-1}\left(Y_{j}-X_{j \beta} d p_{\beta}\right)+ \\
& \left(Y_{i}-X_{i \beta} d p_{\beta}\right) C_{i j}^{-1} X_{j \alpha}=0
\end{aligned}
$$

which is symmetric in $i$ and $j$, and hence the two terms can be written combined as

$$
X_{i \alpha} C_{i j}^{-1}\left(Y_{j}-X_{j \beta} d p_{\beta}\right)=0
$$

and the difference between the best fit and the fiducial model is simply

$$
d p_{\beta}=\left(X_{i \alpha} C_{i j}^{-1} X_{j \beta}\right)^{-1}\left(X_{i \alpha} C_{i j}^{-1} Y_{j}\right) .
$$

To check these analytical calculations and to eliminate potential numerical errors from matrix inversion, we also directly minimized eq. (13) using the numerical scipy routine "optimize.minimize". 8 These numerically identified best-fits were nearly indistinguishable from the analytical calculations above. For convenience and to keep computational costs to a minimum, we used the analytic approach in our main calculations.

The same fitting procedure was performed using the power spectrum, by simply replacing the peak count $N_{i}$ with the power spectrum $P_{i}=P\left(\ell_{i}\right)$ in the above equations. In the case of the power spectrum model (as for the peaks), we used the covariance matrix derived using noisy maps, to include the higher power at small $\ell$ induced by the galaxy shape noise. However, to measure the power

\footnotetext{
8 http://scipy.org/
} 
spectrum derivatives with respect to cosmological parameters, we computed $d P$ using the noiseless maps directly (since noise adds linearly). We choose to use the noisy maps directly, but only with $100<\ell<20$, 000, as cutting off at $\ell=20,000$ (corresponding to $\sim 1$ arcmin) is equivalent to smoothing but has the advantage of faster computation.

The above procedure, applied to each of the 1,000 individual "bias" maps, returns a set of 1,000 best-fit parameters for each specific value of $s$. We then use the distribution of these best-fits to find the average bias in the cosmology parameters (corresponding to the mean best fit), confidence levels, and the goodness-of-fit values.

\section{RESULTS}

\section{A. Power Spectrum}

The impact of $\mathrm{MB}$ on the power spectrum is illustrated in Fig. 2. The levels of bias we chose to show are $s=0.2$, 0.4 and 0.8 . The value $s=0.2$ is close to that expected in LSST; $s=0.4$ is the special case when MB effect disappears completely $(q \equiv 5 s-2=0)$; and $s=0.8$ corresponds to $q=1$ in [20], close to the value expected in the presence of an additional size bias. For comparison, we also show the impact on $P(\ell)$ of varying each cosmological parameter.

For $s \approx 0.2$, the observations suffer a negative bias magnitude of $q=-1.0$. In this case, the effect of diluting a patch of sky wins over the number density increase due to magnification. At all $\ell$ bins, the power is reduced, as the result of the decreasing $\kappa$ fluctuations. For $s=0.4$, we have $q=0$ and expect the MB effect to be absent. This is verified by the lack of any difference between the power spectrum in the $s=0.4$ and the fiducial (unbiased) models, and merely serves as a test of our numerical code. For $s=0.8$, the power is increased on all scales; this behavior has the opposite sign of the $s=0.2$ case, and is consistent with the expectations from $q=5 s-2=$ $2>0$. For cross-check, we calculate $\Delta P / P$ for $s=0.8$ using shear maps. Our results (Fig. 3) are very close to the ones obtained by ref. [20] (their Fig. 1) in the range $1,000<\ell<10,000$ (note that our $s=0.8$ case is equivalent to their $q=1$ case, as they also included the reduced shear correction). However, we noticed that the amplitude of $\Delta P / P$ is 10 times smaller than if we use convergence maps (as in this work).

\section{B. Peak Counts}

Fig. 4 shows the impact of MB on peak counts. For the pure $\mathrm{MB}$ case of $s=0.2$, the height of any positive $\kappa$ peak is reduced due to the negative overall bias. The case $s=0.4$ continues to show no effect from MB. Finally, for the $\mathrm{MB}+\mathrm{SB}$ case of $s=0.8$ (or $q=5 s-2=2$ ), all $\kappa$
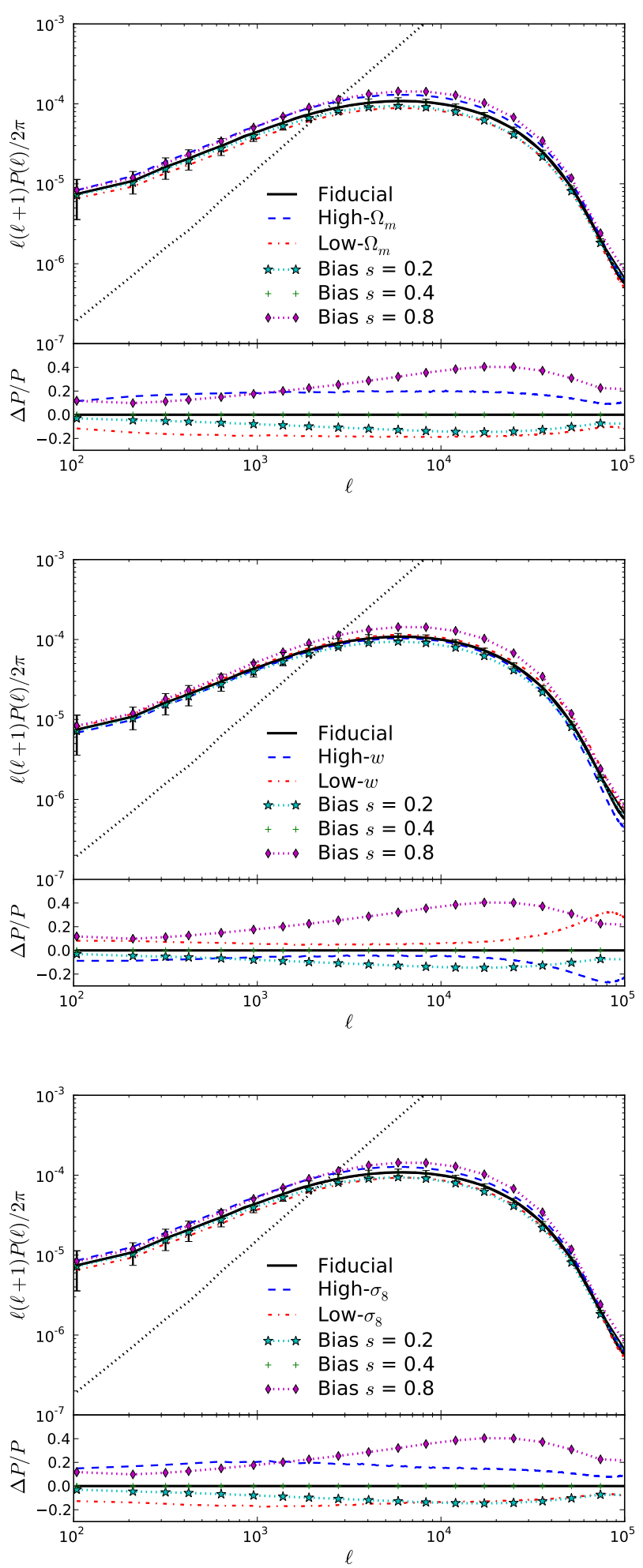

FIG. 2: Changes in the convergence power spectrum caused by magnification bias, as well as by varying individual cosmological parameters. Three levels of bias on the fiducial model are shown with $s=0.2,0.4$ and 0.8 . From top to bottom, besides the fiducial model, we also show changes due to variations in $\Omega_{m}$ (top), $w$ (middle) and $\sigma_{8}$ (bottom). Error bars are for a $12 \mathrm{deg}^{2}$ sky; we expect them to decrease by a factor of $\sim 40$ after scaling the results to LSST's 20,000 $\mathrm{deg}^{2}$ survey. The black dotted line is the galaxy noise for $n_{\text {gal }}=30$ $\operatorname{arcmin}^{2}$. 


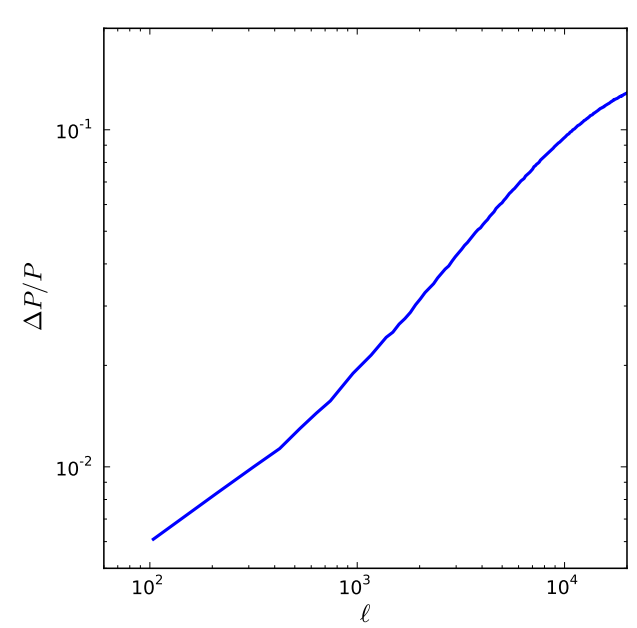

FIG. 3: Fractional difference of shear power due to bias $(s=0.8)$. The slope and values of this curve are very close to the ones obtained by ref. 20] (their Fig. 1) in the range $1,000<\ell<10,000$. Our $s=0.8$ case is equivalent to their $q=1$ case, as they also included the reduced shear correction.

peaks are boosted to a higher value, and consequently the whole distribution is shifted toward the right. The peak counts change in a direction opposite to the $s=0.2$ case, and with a larger amplitude, as expected. We note that for this large positive bias, the abundance of the $\gtrsim 3 \sigma$ (or $\kappa \gtrsim 0.06$ ) peaks increases (as discussed in ref. [22]), but the number of the low peaks is reduced.

A positive MB effect $(s>0.4)$ also reduces the total number of peaks (the number in brackets in Fig. 4). By directly comparing an example of the "bias" maps against its original "true" version, we found that out of the $\sim 3600$ peaks in total, $\sim 120$ peaks disappeared after $\mathrm{MB}$, while only $\sim 60$ new peaks were created. By visual examination of the maps, we found that peak disappearance and creation tends to happen in complex regions, where many peaks are interconnected through filamentlike structures. As an illustration of this, in Fig. 5 we show a typical "high" peak. The shape of this peak is fairly round, likely due to one single massive halo. High peaks like this normally remain a peak after MB. In contrast, Fig. 6 shows a typical low peak that disappears after MB is applied. The original low peak merges into the neighboring, somewhat higher-amplitude peak at the lower left corner - this can be attributed to the lensing bias creating a "ridge" between the two original peaks. The opposite phenomenon happens when the overall MB is negative $(s<0.4)$, where we see an increase in total number of peaks, due to the bias "destroying" ridges and causing a net increase in the number of low peaks.

We have found that MB results in a monotonic increase or decrease for all $\kappa$ peaks before smoothing, depending on the sign of $5 s-2$. Fig. 7 shows the change in $\kappa$ values for all individual pixels, as well as for the peaks, for the
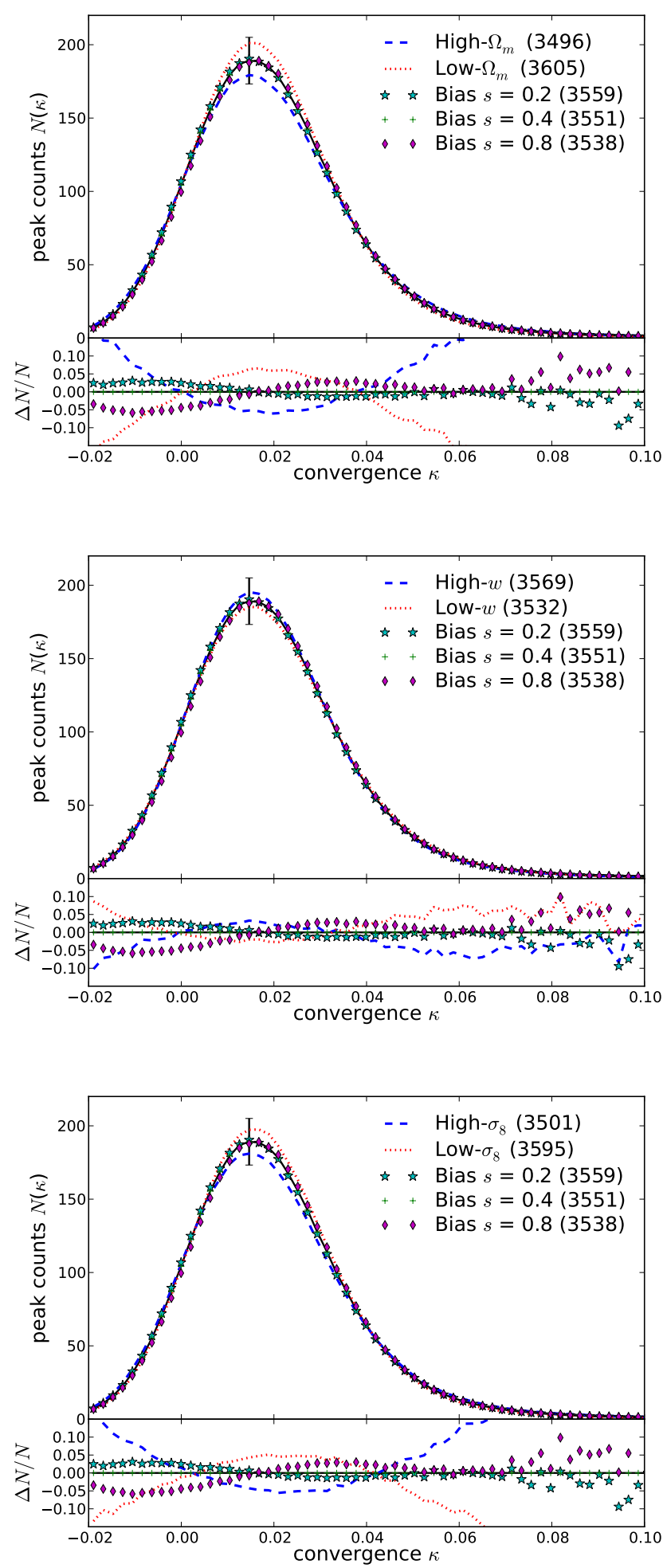

FIG. 4: Peak count changes due to varying levels of magnification bias, as well as due to varying cosmological parameters. Three levels of bias on the fiducial model are shown with $s=0.2,0.4$ and 0.8. As in Fig. 2, we also show changes due to variations in $\Omega_{m}, w$, and $\sigma_{8}$. The number in brackets is the total number of peaks. One error bar is shown to represent a typical error size for $12 \mathrm{deg}^{2}$ sky; we expect this to decrease by a factor of $\sim 40$ after scaling to an LSST-like survey. 


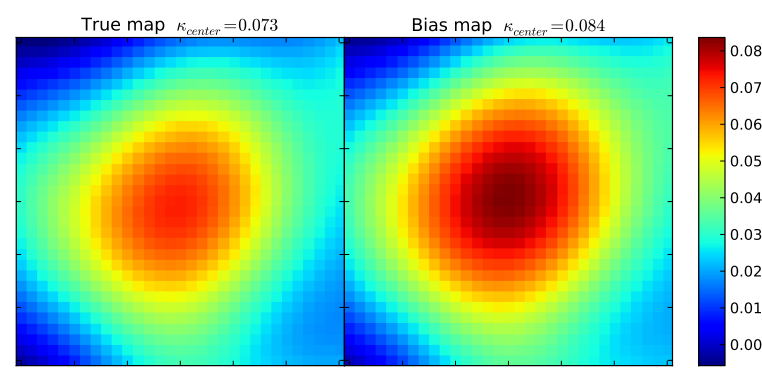

FIG. 5: An example of a high peak (central pixel of the true map at left panel) for $s=1.5$. Most high peaks are characterized by their relatively round shape, due to one single massive halo. After a positive magnification bias is applied to the map (right panel), the peak remains, and with a higher $\kappa$ value.

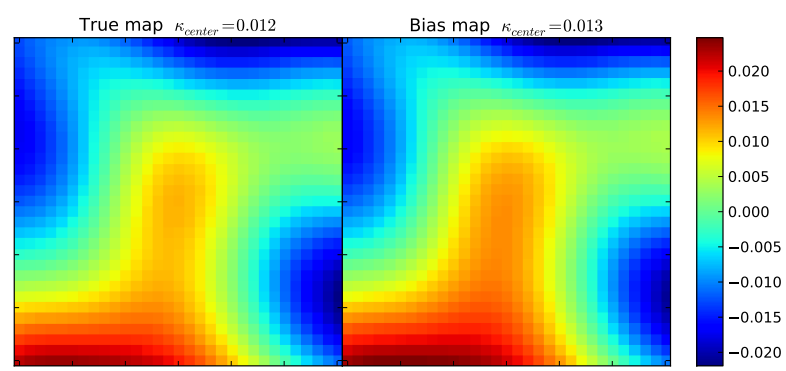

FIG. 6: An example of a typical low peak (central pixel of the true map at left panel) that disappears when a positive magnification bias is applied with $s=1.5$. Such peaks are normally found to be adjacent to another peak with a somewhat higher height, or between multiple higher peaks. After magnification bias (right panel), this particular low peak merges, through a "ridge", with its neighboring peak.

$s=1.5$ case. The peaks that survived the MB (the pixels that are peaks in both "true" and "bias" maps) tend to have a smaller increase in their $\kappa$ value than other random pixels. We speculate that these are the local dominating peaks that could not gain a higher value due to the lack of higher peaks around them. Interestingly, Fig. 7 also show a clear cutoff at $\kappa \lesssim-0.03$ below which no peaks are seen. ${ }^{9}$

Fig. 4 shows that the changes caused by variations in cosmological parameters tend to be more symmetric in the two wings of the peak distribution. For example, at $w=-0.8$, we see fewer high $-\kappa$ peaks, as well as fewer low $-\kappa$ peaks. This shows that no single cosmological pa-

9 This $\kappa_{\min }$ could potentially be a cosmological probe, in analogy with the cosmology-dependent minimum in the probability distribution of $\kappa$ in random directions on the sky [56].

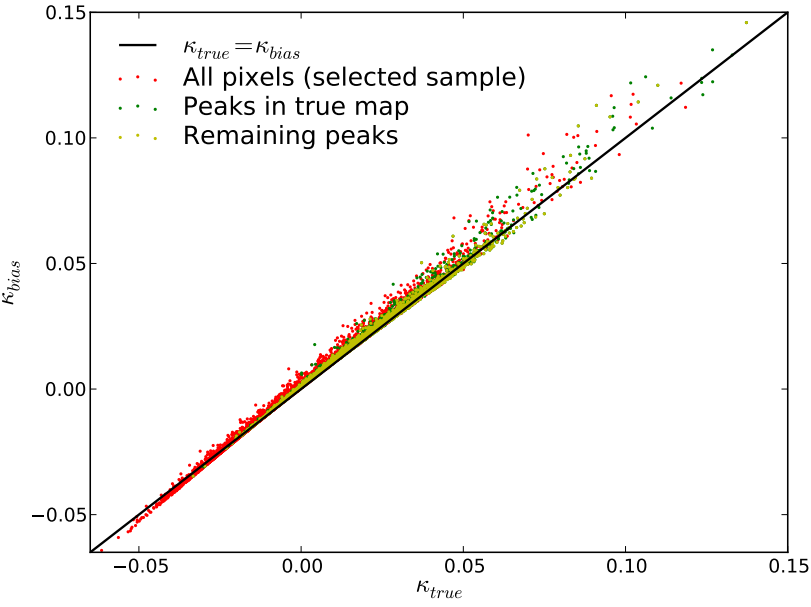

FIG. 7: Comparison of $\kappa_{\text {true }}$ and $\kappa_{\text {bias }}$ on a pixel-by-pixel basis in the $s=1.5$ case. A random subset (10,000 pixels) of all $2048 \times 2048$ pixels (red dots), pixels that are peaks in "true" maps (green dots) and the pixels that remain peaks in the "bias" maps (yellow dots) are shown. Most positive pixels are boosted to a higher value. The true peaks that remain peaks in the biased map tend to have smaller increases in $\kappa$ than a random pixel. This can be attributed to the fact that most such "survivor" peaks are more dominant - i.e. stand out more in their local environment within a smoothing scale.

rameter can mimic the changes caused by MB - however, a linear combination of the three parameters may still resemble such change and can be degenerate with the effects of MB (as we will see below).

Examining the changes due to $\Omega_{m}$ and $\sigma_{8}$, we see a clear degeneracy between these two parameters. This previously known issue (e.g. [25, 27, 57, 58]) is similar to that from cluster counts - both $\Omega_{m}$ and $\sigma_{8}$ can change the number of massive halos; therefore, we can obtain the same number of massive halos (hence the same peak distribution) for a higher value of $\sigma_{8}$, as long as we decrease $\Omega_{m}$. A product of the two parameters in the form of $\Omega_{m} \sigma_{8}^{\gamma}$ is much more tightly constrained by a fixed number of halos. The value of $\gamma$ depends on the relevant mass scale being measured, and varies from 0.3 to 0.6 [6, 57, 59 61]. From our error ellipse, we found $\gamma=0.62$ for the power spectrum and $\gamma=0.48$ for peak counts, by minimizing $\Delta \sigma_{8} / \sigma_{8}+\gamma \Delta \Omega_{m} / \Omega_{m}$ for the 1,000 fitted fiducial maps $\left(\Delta \sigma_{8}\right.$ and $\Delta \Omega_{m}$ are the differences between the fitted values for an individual map and the fiducial parameters).

\section{Cosmological Parameters}

We are now ready to show that without taking into account the effect of magnification bias, WL surveys can deliver cosmological parameters that are biased from the true values by many times their statistical error $\sigma-$ for 


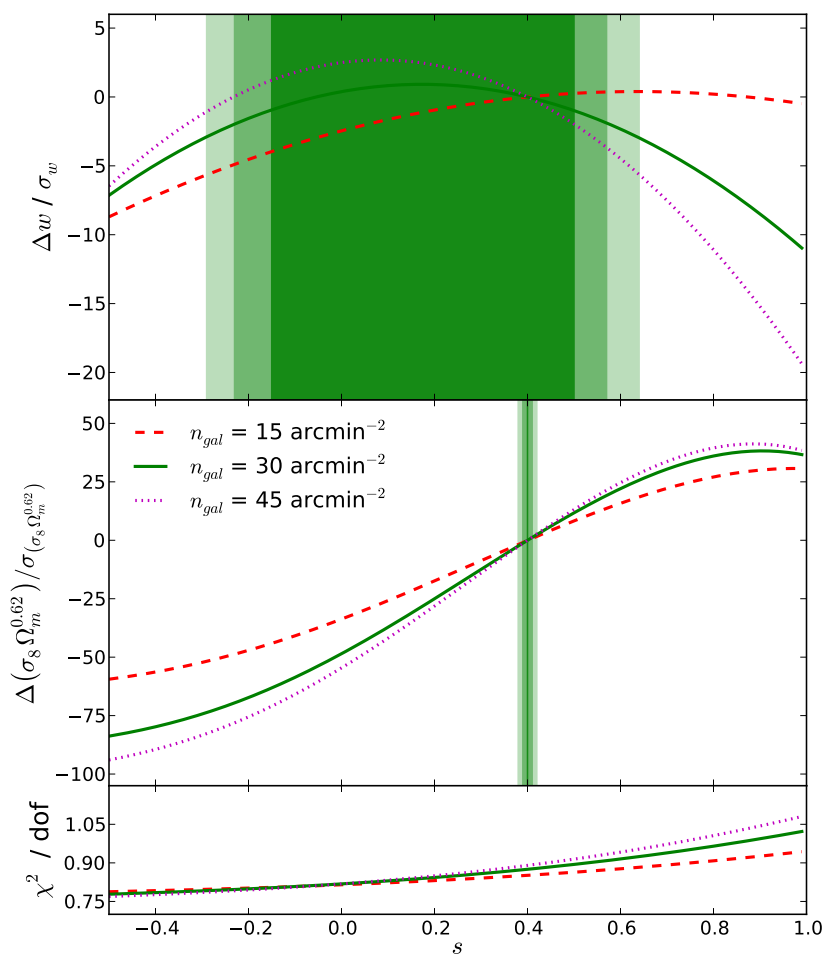

FIG. 8: The biases in cosmological parameters inferred from the power spectrum, in units of their standard deviation $\left(\sigma_{w}=0.016, \sigma_{\left(\sigma_{8} \Omega_{m}^{0.62}\right)}=0.0007\right)$. The shaded regions indicate values of $s$ where the cosmology bias is within 1,2, and $3 \sigma$ (dark to light) for $n_{\text {gal }}=30 \mathrm{arcmin}^{-2}$ (LSST's expected galaxy surface density). In the case of pure $\mathrm{MB}(s=0.2)$ and $\mathrm{MB}+\mathrm{SB}(s=0.8)$ for LSST, $w$ is biased by $0.9 \sigma$ and $-6.1 \sigma$, respectively. The best-constrained combination of $\sigma_{8} \Omega_{m}^{0.62}$ is biased by more than $20 \sigma$ in both cases. The error-bar $\sigma$ has been scaled from our simulation $\left(12 \mathrm{deg}^{2}\right)$ to LSST's planned sky coverage of $20,000 \mathrm{deg}^{2}$.

both the power spectrum and peak counts.

Fig. 8 shows the average deviation of fitted parameters using the power spectrum, in units of their standard deviation $\left(\sigma_{w}=0.016, \sigma_{\left(\sigma_{8} \Omega_{m}^{0.62}\right)}=0.0007\right)$. We have computed this cosmology bias for the range of $-0.5 \leq s \leq 1.0$. The standard deviation is calculated over the 1,000 fiducial maps. Each fitted parameter is marginalized over the other parameters, and scaled from our simulation $\left(12 \mathrm{deg}^{2}\right)$ to LSST's planned sky coverage of 20,000 $\mathrm{deg}^{2}$. The shaded region indicates the values of $s$ where the deviation of the derived parameter is within $1 \sigma, 2 \sigma$ and $3 \sigma$ (dark to light), for galaxy density $n_{\text {gal }}=30 \operatorname{arcmin}^{-2}$. For $s=0.2, \Delta w / \sigma_{w}=0.9$ (although interestingly, as shown in the figure, the bias is not monotonic in $s$ ) and $\Delta\left(\sigma_{8} \Omega_{m}^{0.62}\right) / \sigma_{\left(\sigma_{8} \Omega_{m}^{0.62}\right)}=-25.0$ at $1 \sigma$. We choose to plot $\sigma_{8} \Omega_{m}^{0.62}$ instead of $\sigma_{8}$ and $\Omega_{m}$ individually, because the former is much more tightly con-

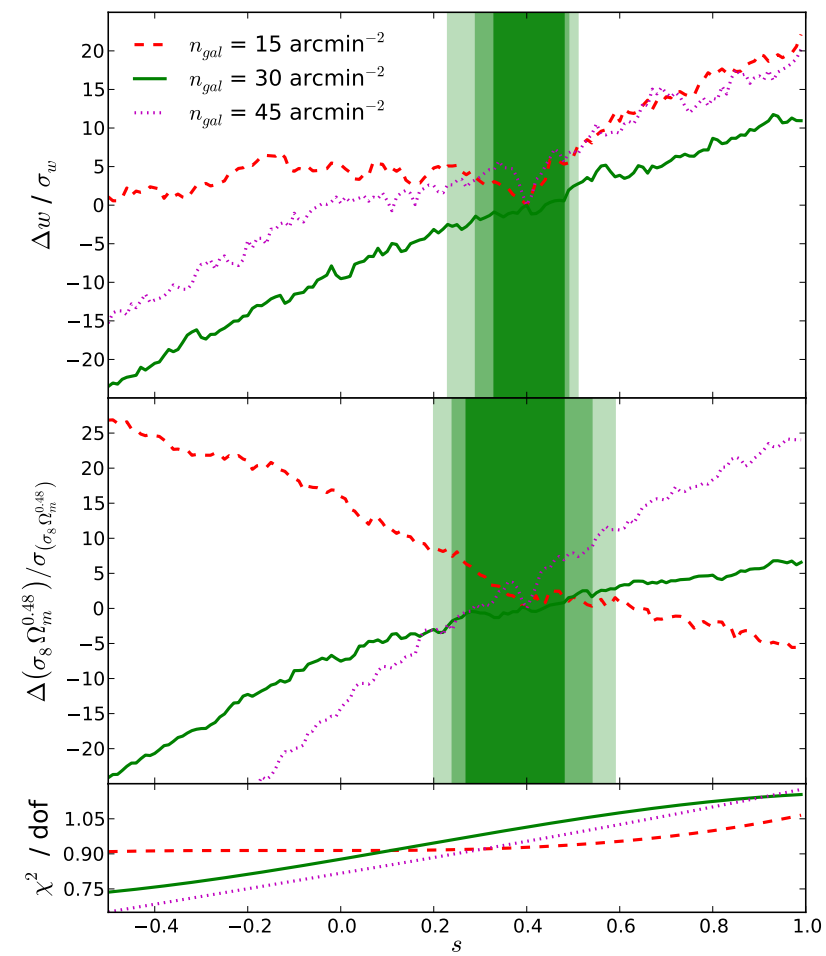

FIG. 9: The biases in cosmological parameters inferred from the peak counts, in units of their standard deviation $\left(\sigma_{w}=0.006, \sigma_{\left(\sigma_{8} \Omega_{m}^{0.48}\right)}=0.0004\right)$. The shaded regions indicate values of $s$ where the cosmology bias is within 1,2 , and $3 \sigma$ (dark to light) for $n_{g a l}=30 \operatorname{arcmin}^{-2}$ (LSST's expected galaxy surface density). $w$ is biased by $-3.1 \sigma(s=0.2)$ and $8.7 \sigma(s=0.8)$, and the combination $\sigma_{8} \Omega_{m}^{0.48}$ by $-3.0 \sigma$ $(s=0.2)$ and $4.7 \sigma(s=0.8)$. The error-bar $\sigma$ has been scaled from our simulation $\left(12 \mathrm{deg}^{2}\right)$ to LSST's planned sky coverage of $20,000 \mathrm{deg}^{2}$.

strained, as discussed in $\S$ IVB.

In Fig. 9, we show the deviations of cosmological parameters inferred from the peak counts $\left(\sigma_{w}=\right.$ $\left.0.006, \sigma_{\left(\sigma_{8} \Omega_{m}^{0.48}\right)}=0.0004\right)$. For $s=0.2$, we find $\Delta w / \sigma_{w}=-3.1, \sim 3$ times larger in magnitude than from the power spectrum; $\Delta\left(\sigma_{8} \Omega_{m}^{0.48}\right) / \sigma_{\left(\sigma_{8} \Omega_{m}^{0.48}\right)}=-3.0$ at $1 \sigma$, which, on the other hand, is much lower than from the power spectrum. For $s=0.8$, we see deviations at similar magnitude but in opposite directions to the $s=0.2$ case, for both the power spectrum and peak counts.

The biases are again shown in two dimensions in Fig. 10, where the Monte Carlo error ellipses, enclosing $68 \%$ of the best-fits, are explicitly shown for the fiducial unbiased maps and biased maps $(s=0.2,0.8)$. In conclusion, WL observations in a survey as large as LSST will need to take MB into account, by including it in the modeling when fitting the observations. Combining information from both the power spectrum and the peak 


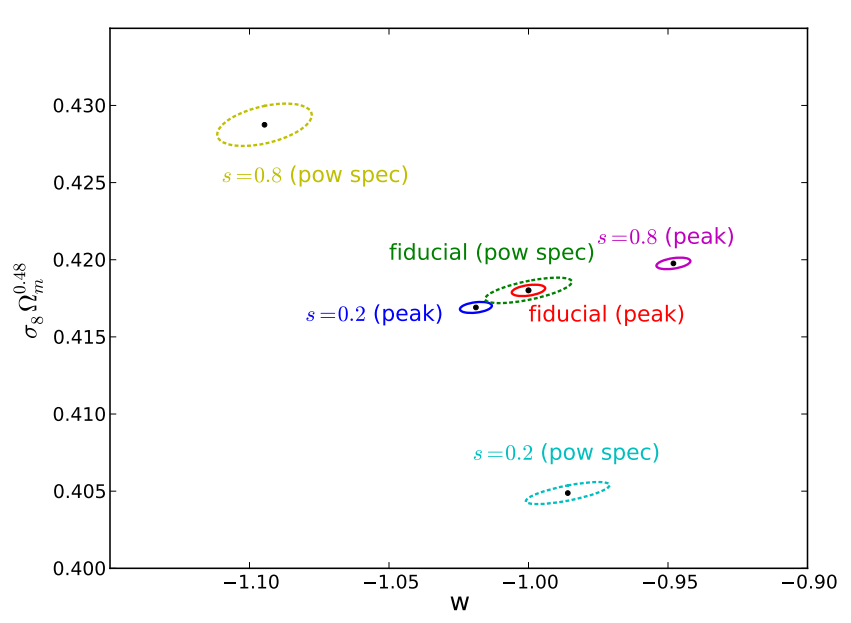

FIG. 10: Error ellipses for the fiducial (unbiased) maps and in the case of magnification bias with $s=0.2$ and 0.8 , for both the power spectrum (using $100<\ell<20,000$ ) and the peak counts (with 200 convergence bins and smoothing scale $1 / \sqrt{2}$ arcmin). Error ellipses contain $68 \%$ of the best-fits, and have been scaled to LSST's sky coverage of $20,000 \mathrm{deg}^{2}$.

counts will be useful, as these two observables are impacted by $\mathrm{MB}$ in different ways, and their combination can help mitigate the biases. The value of $s$ (or other parameters describing higher-order lensing corrections) could be potentially additional parameters in a fitting procedure, simultaneously with the cosmological parameters. We expect that MB has a smaller impact on the current surveys, mainly due to their smaller sky coverage (e.g. COSMOS: $\approx 2 \mathrm{deg}^{2}$, CFHTLenS: $150 \mathrm{deg}^{2}$ ). After scaling $\sigma$ by their sky coverage, we found the deviations to be of order $\sim 0.01 \sigma$ for COSMOS, and $\sim 0.1 \sigma$ for CFHTLenS.

The observed galaxy number density will also affect the level of MB. In Figures 8 and 9, we also show the parameter biases for $n_{\text {gal }}=15$ and $45 \operatorname{arcmin}^{-2}$. For the power spectrum, the slope near $s=0.4$ tends to be steeper for larger $n_{\text {gal }}$. This means deeper surveys with higher galaxy number densities (hence smaller galaxy noise) are more sensitive to MB when galaxy noise (eq. 8) is smaller. For peak counts, MB impacts the derived $\sigma_{8} \Omega_{m}^{0.48}$ for shallower surveys $\left(n_{\text {gal }}=15\right.$ arcmin $)$ in opposite direction to surveys with higher number density.

\section{DISCUSSIONS}

In this work, we made several assumptions and simplifications, which we must highlight here:

(i) We assumed the number of peaks and the power spectrum depends linearly on cosmology. For example, in our analysis, we used "forward" derivatives for $d \bar{N} / d p$, built with the fiducial and the three "high" cosmologies from Table II for a finite difference. We can also use

\begin{tabular}{|c|c|c|c|c|}
\hline & \multicolumn{2}{|c|}{$s=0.2$} & \multicolumn{2}{|c|}{$s=0.8$} \\
\hline power spectrum & forward & backward & forward & backward \\
\hline$\Delta \Omega_{m} / \sigma_{\Omega_{m}}$ & -21.0 & -23.2 & 61.6 & 67.0 \\
\hline$\Delta w / \sigma_{w}$ & 0.9 & 0.4 & -6.1 & -4.1 \\
\hline$\Delta \sigma_{8} / \sigma_{\sigma_{8}}$ & 7.6 & 8.6 & -28.3 & -30.9 \\
\hline$\Delta\left(\sigma_{8} \Omega_{m}^{0.62}\right) / \sigma_{\left(\sigma_{8} \Omega_{m}^{0.62}\right)}$ & -25.0 & -27.3 & 36.0 & 37.4 \\
\hline peak counts & forward & backward & forward & backward \\
\hline$\Delta \Omega_{m} / \sigma_{\Omega_{m}}$ & 1.4 & 1.5 & -2.9 & -3.2 \\
\hline$\Delta w / \sigma_{w}$ & -3.1 & -4.4 & 8.7 & 4.5 \\
\hline$\Delta \sigma_{8} / \sigma_{\sigma_{8}}$ & -2.6 & -2.7 & 4.8 & 3.1 \\
\hline$\Delta\left(\sigma_{8} \Omega_{m}^{0.48}\right) / \sigma_{\left(\sigma_{8} \Omega_{m}^{0.48}\right)}$ & -3.0 & -3.1 & 4.7 & 0.2 \\
\hline
\end{tabular}

TABLE III: Deviations of cosmological parameters evaluated at $s=0.2$ (MB only) and $s=0.8(\mathrm{MB}+\mathrm{SB})$. Results from "forward" and "backward" derivatives are compared side-byside.

the three "low" cosmologies to obtain "backward" finitedifference derivatives. When we do so, we find the resulting deviations to have similar magnitude (Table III) to the ones from "forward" derivatives, except for a significantly lower value for $\Delta \sigma_{8} \Omega_{m}^{0.48}$ for peak counts.

We also attempted to use a spline interpolation, using all three data-points for each parameter to describe the cosmology-dependence. This enables us to utilize all 7 cosmologies simultaneously, but we lose the advantage of the analytical method to obtain the best-fits (eq. 20). We used the numerical method to find the bestfits with spline interpolation, and found mean biases similar to those from linear interpolations. However, the error ellipses from spline interpolation were considerably smaller, and suspiciously coincident with our simulated range of model parameters. This is likely due to the spline tails that curve dramatically outside our parameter region, and hence artificially force the fit to stay within our simulated range of model parameters for each map. To solve this issue, we will need to have a larger grid of simulation parameters, which will also help us understand the dependence of peak counts on cosmology more accurately.

(ii) We used convergence maps only at a single redshift. This is motivated by the fact that the effect of MB depends weakly on $z$. At low redshift, $s$ is mainly dependent on the slope of this power-law tail, and MB will have similar level of impact for all galaxies. For galaxies at higher redshift, $m_{\text {lim }}$, when redshifted to the rest frame of the galaxy, moves closer to the exponential part of the luminosity function, so we expect $s$ to increase to a larger value. A redshift-dependent correction to MB that folds in the correct $z$-distribution of high- $z$ galaxies will eventually be necessary.

(iii) We ignored all instrumental and measurement errors. In reality, the point spread function (PSF) deconvolution and the measurement of galaxy shapes accurately is a difficult task, and has received thorough discussions (e.g. 62, 63]). Ref. [33, used simulated shear maps with realistic galaxy properties and has taken into account distortions from both the atmosphere and optical errors expected for LSST. They have shown that, though peak sig- 
nificance is reduced, the addition of these errors does not significantly degrade the cosmological constraints, compared to considering shape noise only. While our basic conclusion, that MB is significant, likely remains valid in the presence of such errors, the detailed modeling of MB will need to incorporate these additional sources of error.

(iv) In this paper, we choose to work with convergence maps, as they are computationally simpler. Using galaxies with sizes larger than the PSF, the convergence field can potentially be inferred by combining galaxy size and flux measurements, as lensing modifies these two quantities by different factors of $1+\kappa$ and $1+2 \kappa$, respectively, in the weak lensing limit 64 67]. In current practice, reduced shear maps are obtained by measuring the shapes of individual galaxies. The observer can deduce the aperture mass $\left(M_{a p}\right)$, a smoothed form of convergence, by applying a convolution over tangential components of shear 68. Ref. 31 has shown that both shear and convergence statistics give similar constraints when compared at the same scale, but once again, the impact of lensing bias should be modeled directly on the shear field.

(v) Although WL surveys may implement a sharp flux cut, the size bias is likely to be more complicated, with an effective weighting on galaxies that depends monotonically on their size, but in a gradual fashion, rather than a step function. In the idealized case of a sharp size cut, our analysis remains applicable, with a suitable re-interpretation of $5 s$ as a stand-in for $5 s+\beta$, where $\beta$ is the logarithmic slope of the size distribution (at the size cut). In this simplified case, the bias induced by the size cut is likely larger than the one induced by the flux cut. For example, 21] showed that, for a survey with magnitude cut $i_{A B}=24$ and size cut $r=1.2$ ", the impact of MB becomes positive and $q=5 s+\beta-2 \sim 1-2$, which is equivalent to our cases with $s=0.6-0.8$. From Fig. 10 , we see the derived parameters remain many $\sigma$ away from the true parameters, but in the opposite direction. This demonstrates that size cut is likely to be important, and also that it is necessary in future work to investigate the effects of size bias in more detail.

(vi) We have tested the impact of $\mathrm{MB}$ on three parameters, $\Omega_{m}, w$ and $\sigma_{8}$. When additional cosmological parameters are considered (e.g. $\Omega_{b}, H_{0}, n_{s}, w_{a}$ ), the impact of $\mathrm{MB}$ may be more severe, since a combination involving the new parameters could mimic the MB better. In order to test this, we need to run more N-body simulations with other parameters varying to build a more complete cosmological model.

(vii) Optimizing the number of bins has not been the focus of this work. However, the choice of the number of bins has an effect on the error sizes. As shown in Fig. 11, for peak counts, the values of derived parameters and marginalized errors only converge at $\gtrsim 150$ bins. Once the number of bins exceeds this value, we see a roughly constant plateau extending to $\gtrsim 500$ bins (beyond which the results become unreliable, due to having too few realizations of maps and the sample covariance matrix becoming singular near 1000 bins). Therefore, we chose to
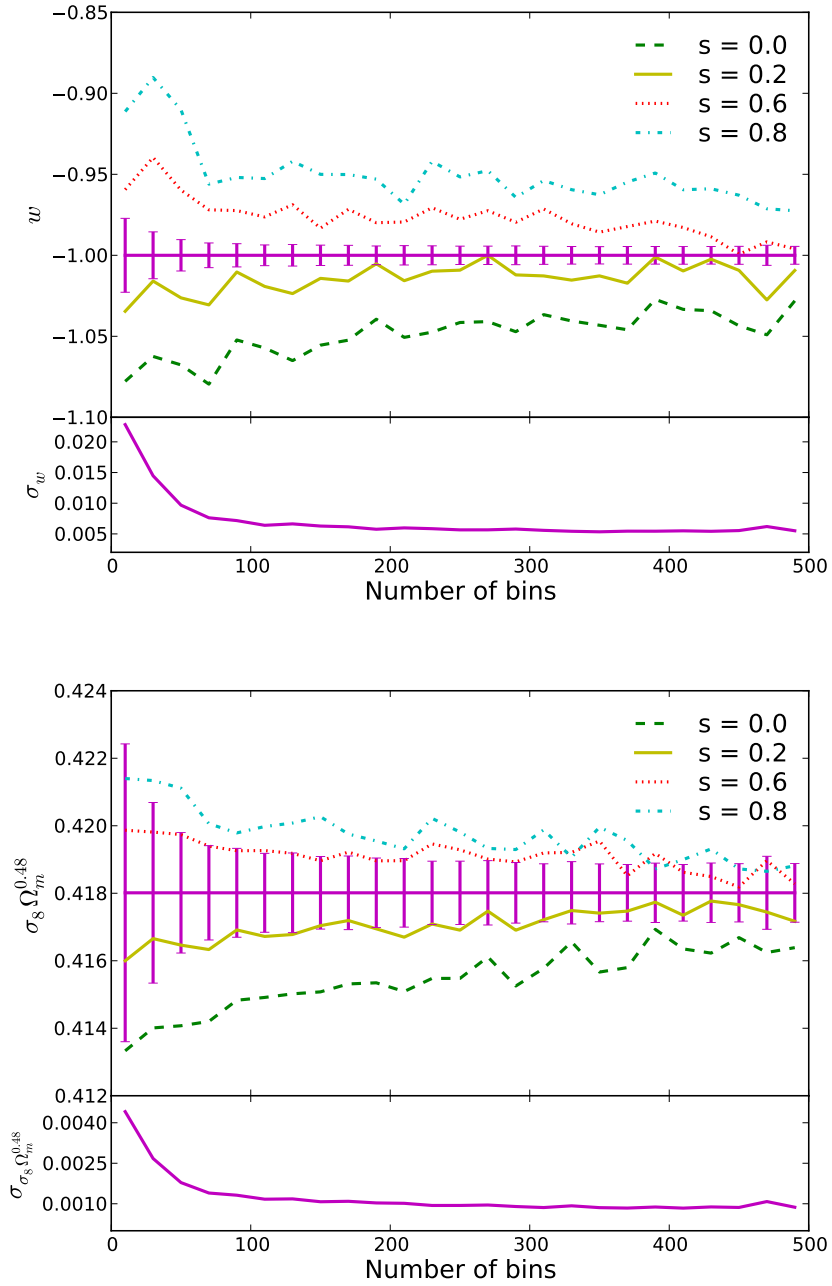

FIG. 11: The derived cosmological parameters and marginalized error for each parameter as a function of the number of bins, using peak counts. The error sizes have been scaled to LSST's sky coverage of $20,000 \mathrm{deg}^{2}$. The error sizes tend to decrease with larger number of bins. The results for $\gtrsim 500$ bins are unreliable due to the limited number of realizations in our simulation suite.

use 200 bins in this work.

\section{SUMMARY}

In this paper, we have studied the effect of magnification bias on peak statistics, using convergence maps from ray-tracing N-body simulations. Using maps in a suite of simulations, we can predict the convergence power spectrum or peak count distribution as a function of $\Omega_{m}, w$, and $\sigma_{8}$. Using this tool, we found the biases in cosmological parameters, when convergence maps in the fiducial cosmology, modified by magnification bias, were used to find the best-fit cosmology, without taking MB into account in the fits. 
Near the flux limit of future WL survey, such as LSST, the galaxy number counts have a logarithmic slope of $s \approx 0.2$. This causes a bias in the inferred value of $w$ by $0.9 \sigma$ and of $\sigma_{8} \Omega_{m}^{0.62}$ by $-25.0 \sigma$ when using the power spectrum, and by $-3.1 \sigma$ for $w$ and $-3.1 \sigma$ for $\sigma_{8} \Omega_{m}^{0.48}$ when using peak counts. These results are scaled to WL observations expected from LSST. However, for recent surveys, such as COSMOS and CFHTLenS, the deviations are generally negligible $(\sim 0.01 \sigma$ and $\sim 0.1 \sigma$, respectively), due to their smaller sky coverage.

We conclude that it is necessary that cosmological simulations consider MB effects, when they are used to match observations. We have found that $w$ inferred from the power spectrum is less impacted by MB, but peak count is a less biased method to infer $\sigma_{8} \Omega_{m}^{\gamma}$. Future work on magnification biases should incorporate the many improvements we have emphasized that are necessary, including (i) the redshift-dependence of the bias, (ii) the impact on shear maps with realistic measurement errors and the peak statistics derived from these maps, (iii) more complex biases induced by the size-dependent measurement errors cut on galaxies, and (iv) additionally, the potential of using magnification bias and size bias as a signal to tighten the constraints on convergence field 64 67. Our results suggest that lensing biases can be mitigated by combining the power spectrum and the peak counts, which produce biases in very different directions in cosmological parameter space.

\section{Acknowledgments}

We thank Kevin Huffenberger for useful discussions. This research utilized resources at the New York Center for Computational Sciences, a cooperative effort between Brookhaven National Laboratory and Stony Brook University, supported in part by the State of New York. This work is supported in part by the U.S. Department of Energy under Contract No. DE-AC02-98CH10886 and by the NSF under grant AST-1210877. The simulations were created on the IBM Blue Gene/L and /P New York Blue computer and the maps were created analyzed on the LSST/Astro Linux cluster at BNL.
[1] A. Refregier, ARA\&A 41, 645 (2003), [arXiv:arXiv:astro-ph/0307212].

[2] P. Schneider, ArXiv Astrophysics e-prints (2005), [arXiv:arXiv:astro-ph/0509252].

[3] H. Hoekstra and B. Jain, Annual Review of Nuclear and Particle Science 58, 99 (2008), arXiv:0805.0139.

[4] M. Bartelmann, Classical and Quantum Gravity 27, 233001 (2010).

[5] T. Schrabback et al., A\&A 516, A63 (2010), arXiv:0911.0053.

[6] M. Kilbinger et al., MNRAS 430, 2200 (2013), arXiv:1212.3338.

[7] R. L. Webster, P. C. Hewett, M. E. Harding and G. A. Wegner, Nature 336, 358 (1988).

[8] W. Fugmann, A\&A 204, 73 (1988).

[9] R. Narayan, ApJL 339, L53 (1989).

[10] P. Schneider, A\&A 221, 221 (1989).

[11] J. Verner Villumsen, ArXiv Astrophysics e-prints (1995), [arXiv:arXiv:astro-ph/9512001].

[12] J. V. Villumsen, W. Freudling and L. N. da Costa, ApJ 481, 578 (1997), [arXiv:arXiv:astro-ph/9606084].

[13] R. Moessner, B. Jain and J. V. Villumsen, MNRAS 294, 291 (1998), [arXiv:arXiv:astro-ph/9708271].

[14] N. Kaiser, ApJ 498, 26 (1998), [arXiv:arXiv:astro$\mathrm{ph} / 9610120]$.

[15] M. Loverde, L. Hui and E. Gaztañaga, Phys. Rev. D 75, 043519 (2007), [arXiv:arXiv:astro-ph/0611539].

[16] T. Matsubara, ApJL 537, L77 (2000), [arXiv:arXiv:astro-ph/0004392].

[17] L. Hui, E. Gaztañaga and M. Loverde, Phys. Rev. D 76, 103502 (2007), arXiv:0706.1071.

[18] L. Hui, E. Gaztañaga and M. Loverde, Phys. Rev. D 77, 063526 (2008), arXiv:0710.4191.

[19] M. Loverde, S. Marnerides, L. Hui, B. Ménard and A. Lidz, Phys. Rev. D 82, 103507 (2010),
arXiv:1004.1165].

[20] F. Schmidt, E. Rozo, S. Dodelson, L. Hui and E. Sheldon, ApJ 702, 593 (2009), arXiv:0904.4703.

[21] F. Schmidt, E. Rozo, S. Dodelson, L. Hui and E. Sheldon, Physical Review Letters 103, 051301 (2009), arXiv:0904.4702.

[22] F. Schmidt and E. Rozo, ApJ 735, 119 (2011), arXiv:1009.0757.

[23] A. Albrecht et al., arXiv:astro-ph/0609591.

[24] B. Jain and L. Van Waerbeke, ApJL 530, L1 (2000), arXiv:astro-ph/9910459.

[25] J. P. Dietrich and J. Hartlap, MNRAS 402, 1049 (2010), arXiv:0906.3512.

[26] M. Maturi, C. Angrick, F. Pace and M. Bartelmann, A\&A 519, A23 (2010), arXiv:0907.1849.

[27] J. M. Kratochvil, Z. Haiman and M. May, Phys. Rev. D 81, 043519 (2010), arXiv:0907.0486.

[28] X. Yang et al., Phys. Rev. D 84, 043529 (2011), arXiv:1109.6333.

[29] L. Marian, R. E. Smith, S. Hilbert and P. Schneider, MNRAS 423, 1711 (2012), arXiv:1110.4635.

[30] J. M. Kratochvil et al., Phys. Rev. D 85, 103513 (2012), arXiv:1109.6334.

[31] S. Pires, A. Leonard and J.-L. Starck, MNRAS 423, 983 (2012), arXiv:1203.2877.

[32] X. Yang, J. M. Kratochvil, K. Huffenberger, Z. Haiman and M. May, Phys. Rev. D 87, 023511 (2013), arXiv:1210.0608.

[33] D. Bard et al., ApJ 774, 49 (2013), arXiv:1301.0830.

[34] E. L. Turner, J. P. Ostriker and J. R. Gott, III, ApJ 284, 1 (1984).

[35] P. Schechter, ApJ 203, 297 (1976).

[36] A. Gabasch et al., A\&A 421, 41 (2004), [arXiv:arXiv:astro-ph/0403535].

[37] A. Gabasch et al., A\&A 448, 101 (2006), 
[arXiv:arXiv:astro-ph/0510339].

[38] C. Chang et al., ArXiv e-prints (2013), arXiv:1305.0793.

[39] LSST Science Collaboration et al., ArXiv e-prints (2009), arXiv:0912.0201.

[40] R. Laureijs, ArXiv e-prints (2009), arXiv:0912.0914.

[41] N. Scoville et al., ApJS 172, 38 (2007), [arXiv:arXiv:astro-ph/0612306].

[42] C. Heymans et al., MNRAS 427, 146 (2012), arXiv:1210.0032.

[43] The Dark Energy Survey Collaboration, ArXiv Astrophysics e-prints (2005), [arXiv:arXiv:astro-ph/0510346].

[44] A. Refregier, Experimental Astronomy 23, 17 (2009), arXiv:0802.2522

[45] J. T. A. de Jong, G. A. Verdoes Kleijn, K. H. Kuijken and E. A. Valentijn, Experimental Astronomy 35, 25 (2013), arXiv:1206.1254.

[46] M. Takada, Subaru Hyper Suprime-Cam Project, in American Institute of Physics Conference Series, edited by N. Kawai and S. Nagataki, , American Institute of Physics Conference Series Vol. 1279, pp. 120-127, 2010.

[47] E. Komatsu et al., ApJS 192, 18 (2011), arXiv:1001.4538.

[48] A. Lewis, A. Challinor and A. Lasenby, ApJ 538, 473 (2000), [arXiv:arXiv:astro-ph/9911177].

[49] R. Hockney and J. Eastwood, Chap 4, 113 (1988).

[50] Y.-S. Song and L. Knox, Phys. Rev. D 70, 063510 (2004), [arXiv:arXiv:astro-ph/0312175].

[51] L. van Waerbeke, MNRAS 313, 524 (2000), [arXiv:arXiv:astro-ph/9909160].

[52] D. N. Limber, ApJ 117, 134 (1953).
[53] R. E. Smith et al., MNRAS 341, 1311 (2003), [arXiv:arXiv:astro-ph/0207664].

[54] J. Hartlap, P. Simon and P. Schneider, A\&A 464, 399 (2007), [arXiv:arXiv:astro-ph/0608064].

[55] T. W. Anderson, An Introduction to Multivariate Statistical Analysis, third ed. (Wiley, New York, NY, 2003).

[56] E. V. Linder, JCAP 3, 19 (2008), arXiv:0711.0743.

[57] E. Rozo et al., ApJ 708, 645 (2010), arXiv:0902.3702.

[58] S. Basilakos and M. Plionis, ApJL 714, L185 (2010), arXiv:1003.2559.

[59] H. Mantz, K. Jacobs and K. Mecke, Journal of Statistical Mechanics: Theory and Experiment 12, 15 (2008).

[60] J. P. Henry, A. E. Evrard, H. Hoekstra, A. Babul and A. Mahdavi, ApJ 691, 1307 (2009), arXiv:0809.3832.

[61] A. Vikhlinin et al., ApJ 692, 1060 (2009), arXiv:0812.2720.

[62] N. Kaiser, G. Squires and T. Broadhurst, ApJ 449, 460 (1995), [arXiv:arXiv:astro-ph/9411005].

[63] H. Bonnet and Y. Mellier, A\&A 303, 331 (1995).

[64] A. Vallinotto, S. Dodelson and P. Zhang, Phys. Rev. D 84, 103004 (2011), arXiv:1009.5590.

[65] F. Schmidt et al., ApJL 744, L22 (2012), arXiv:1111.3679.

[66] B. Casaponsa et al., MNRAS 430, 2844 (2013), arXiv:1209.1646].

[67] A. Heavens, J. Alsing and A. H. Jaffe, MNRAS 433, L6 (2013), arXiv:1302.1584.

[68] P. Schneider, MNRAS 283, 837 (1996), [arXiv:arXiv:astro-ph/9601039]. 